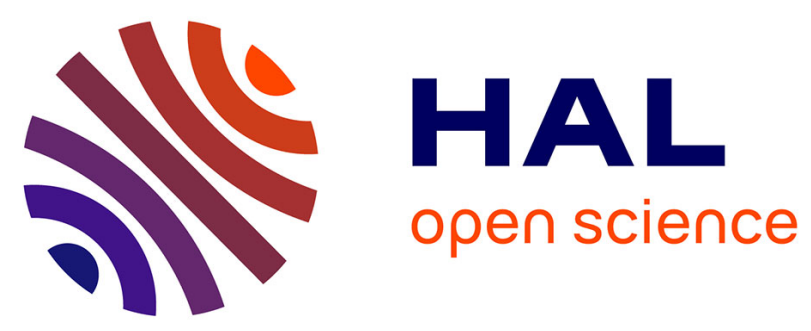

\title{
Near-infrared active polarimetric and multispectral laboratory demonstrator for target detection
}

Mehdi Alouini, François Goudail, Arnaud Grisard, Jérôme Bourderionnet, Daniel Dolfi, Arnaud Bénière, Ivar Baarstad, Trond Løke, Peter Kaspersen, Xavier Normandin, et al.

\section{To cite this version:}

Mehdi Alouini, François Goudail, Arnaud Grisard, Jérôme Bourderionnet, Daniel Dolfi, et al.. Nearinfrared active polarimetric and multispectral laboratory demonstrator for target detection. Applied optics, 2009, 48 (8), pp.1610. 10.1364/AO.48.001610 . hal-00665115

\section{HAL Id: hal-00665115 \\ https://hal.science/hal-00665115}

Submitted on 6 Apr 2012

HAL is a multi-disciplinary open access archive for the deposit and dissemination of scientific research documents, whether they are published or not. The documents may come from teaching and research institutions in France or abroad, or from public or private research centers.
L'archive ouverte pluridisciplinaire HAL, est destinée au dépôt et à la diffusion de documents scientifiques de niveau recherche, publiés ou non, émanant des établissements d'enseignement et de recherche français ou étrangers, des laboratoires publics ou privés. 


\title{
Near-infrared active polarimetric and multispectral laboratory demonstrator for target detection
}

\author{
Mehdi Alouini, ${ }^{1,2, *}$ François Goudail, ${ }^{3}$ Arnaud Grisard, ${ }^{1}$ Jérôme Bourderionnet, ${ }^{1}$ \\ Daniel Dolfi, ${ }^{1}$ Arnaud Bénière, ${ }^{1,3}$ Ivar Baarstad, ${ }^{4}$ Trond Løke, ${ }^{4}$ Peter Kaspersen, ${ }^{4}$ \\ Xavier Normandin, ${ }^{5}$ and Gerard Berginc ${ }^{5}$ \\ ${ }^{1}$ Thales Research \& Technology, RD 128, 91767 Palaiseau, France \\ ${ }^{2}$ Institut de physique de Rennes, CNRS, Université Rennes 1, 35042 Rennes, France \\ ${ }^{3}$ Laboratoire Charles Fabry de l'Institut d'Optique, CNRS, Université Paris-Sud, Campus Polytechnique, \\ RD 128, 91127 Palaiseau, France \\ ${ }^{4}$ Norsk Elektro Optikk AS, Solheimvegen 62A, N-1471 Lørenskog, Norway \\ ${ }^{5}$ Thales Optronique SA, two Av. Gay-Lussac CS 9050278995 Elancourt Cedex, France \\ *Corresponding author: mehdi.alouini@thalesgroup.com
}

Received 12 September 2008; revised 29 January 2009; accepted 5 February 2009; posted 9 February 2009 (Doc. ID 101540); published 9 March 2009

\begin{abstract}
We report on the design and exploitation of a real-field laboratory demonstrator combining active polarimetric and multispectral functions. Its building blocks, including a multiwavelength pulsed optical parametric oscillator at the emission side and a hyperspectral imager with polarimetric capability at the reception side, are described. The results obtained with this demonstrator are illustrated on some examples and discussed. In particular it is found that good detection performances rely on joint use of intensity and polarimetric images, with these images exhibiting complementary signatures in most cases. (C) 2009 Optical Society of America
\end{abstract}

OCIS codes: $\quad 260.5430,100.2960,030.4280,290.5880$.

\section{Introduction}

The increasing capability in terms of image acquisition, data storage, and fast processing as well as the development of new sensors enables modern multidimensional imaging [1,2]. Hence the properties of the light backscattered by a given scene, including wavelength and polarization, can be analyzed and taken into account in order to reveal contrasts that are not seen in common intensity (INT) images [3-10]. Following this approach we have been investigating, in the past few years, the benefits of combining active polarimetric and multispectral imaging [11,12]. These studies have evidenced that, in most cases, single-wavelength polarimetric images do not bring

0003-6935/09/081610-09\$15.00/0

(C) 2009 Optical Society of America enough information to discriminate between objects of different nature. For instance, if one considers a dark diffuse object at a given wavelength, this object will appear bright in the polarimetric image, namely, almost as bright as a metallic object. To overcome this ambiguity it is necessary to acquire another polarimetric image at a second wavelength at which the diffuse object appears bright in the INT image and consequently dark in the polarimetric image [13]. Similarly multispectral and hyperspectral INT images make it possible, in most cases, to discriminate two apparently similar objects through their spectral signature $[14,15]$. However, these images, being less sensitive to the texture of objects than polarimetric images, may fail to detect, for instance, camouflage paints that mimic background spectral signatures.

In this context one could wonder whether the realization of an active imaging system combining both 
polarimetric and multispectral capabilities, having a reasonable size and being scalable to short and midrange applications, is conceivable. This raises the question of the number of wavelengths that must be used for optimized detection, as well as the way of processing the spectropolarimetric images to perform efficient detection of man-made objects.

This paper is divided into two main sections. The first half is devoted to the description of the laboratory demonstrator we have developed. In particular, its main building blocks are detailed, emphasizing the main motivations behind our technical choices. In the second half we illustrate, on some images, the most interesting results we have obtained in terms of target detection. These results are exploited to draw some conclusions, such as the benefits of joint use of polarimetric and INT spectra to improve target detection capability of the system.

\section{Description of the Laboratory Demonstrator}

Before describing the laboratory demonstrator, let us establish the basis of our approach. Polarimetric imaging aims at characterizing the properties of materials with respect to their interaction with polarized light [16-18]. These properties can be exhaustively represented by the Mueller matrix, $M$, which describes how the polarization state of an incident light evolves after interaction with the material. It consists of 16 real-valued coefficients $[19,20]$. However, when observing natural scenes in monostatic configuration, i.e., when the illumination beam is aligned with the line of sight and when the objects are purely depolarizing, the Mueller matrix of most materials becomes diagonal $[21,22]$, that is,

$$
M=\left[\begin{array}{llll}
1 & 0 & 0 & 0 \\
0 & a & 0 & 0 \\
0 & 0 & a & 0 \\
0 & 0 & 0 & b
\end{array}\right]
$$

where $a$ accounts for the linear depolarization of the material, and $b$ accounts for the circular depolarization. When the material is purely depolarizing, it is experimentally shown that $b$ is close to $a$ [23]. The overall depolarization power of the material being given by $(2 a+b) / 3$ shows that $a$ is a good estimation of the material depolarization power. It can be evaluated by (i) shining the material with a linearly polarized light and (ii) analyzing the backscattered light along the parallel $\left(I_{\|}\right)$and crossed $\left(I_{\perp}\right)$ polarizations. One thus has $a=\left(I_{\|}-I_{\perp}\right) /\left(I_{\|}+I_{\perp}\right)$, which corresponds to the orthogonal state contrast (OSC). It is worthwhile to mention that, in the limits of our assumptions, $a$ is also equal to the degree of polarization [24] of the emerging state of light. Hence, if the observed materials are purely depolarizing, the OSC represents the coefficient $a$ of their Mueller matrix as well as the degree of polarization of the backscattered light. If materials are not purely depolarizing, interpretation of the OSC is more complex, but it still conveys information, which can be useful for target detection, for example. The polarimetric images we consider in this paper are formed, for a given wavelength, by pixelwise computing the OSC, namely,

$$
\mathrm{OSC}=\left(I_{\|}-I_{\perp}\right) /\left(I_{\|}+I_{\perp}\right) .
$$

The laboratory imaging system we have designed can be operated in active or passive modes and provides multispectral and polarimetric capabilities. In the passive mode, both INT and polarimetric images can be acquired. Nevertheless, for polarimetric imaging, active illumination of the scene is, in general, preferred, because the state of polarization of the illumination beam can be fixed. By optimizing this polarization state, the polarimetric contrast of the image can be made higher than that obtained with unpolarized ambient light. Furthermore, in the active configuration, the illumination beam is aligned with the line of sight. The polarimetric signature of the imaged object is then better defined and related, at first approximation, to its depolarization capabilities only. Images obtained in this way are thus easier to interpret. In our case the illumination beam is linearly polarized along the vertical direction. A schematic representation of the demonstrator is reported in Fig. 1. It can be divided into four main building blocks: emission part, reception part, polarization analysis part, and control/acquisition part. In Subsections 2.A-2.D, we briefly describe these four building blocks, emphasizing our technical choices.

\section{A. Reception Part}

The reception part of the laboratory imager consists of a customized HySpex SWIR-320 hyperspectral imager developed by Norsk Elektro Optikk (Norway) [25]. As shown in Fig. 2, the incoming light is focused onto a slit, collimated, and then directed to a polarization-independent transmission grating that disperses it. The fore-optics and collimating optics consist of aspheric mirrors to avoid spherical and chromatic aberrations. The dispersed radiation is then imaged on a two-dimensional detector array due to a customized lens system. The position at which the focused light hits the detector is wavelength dependent. Consequently each line of the

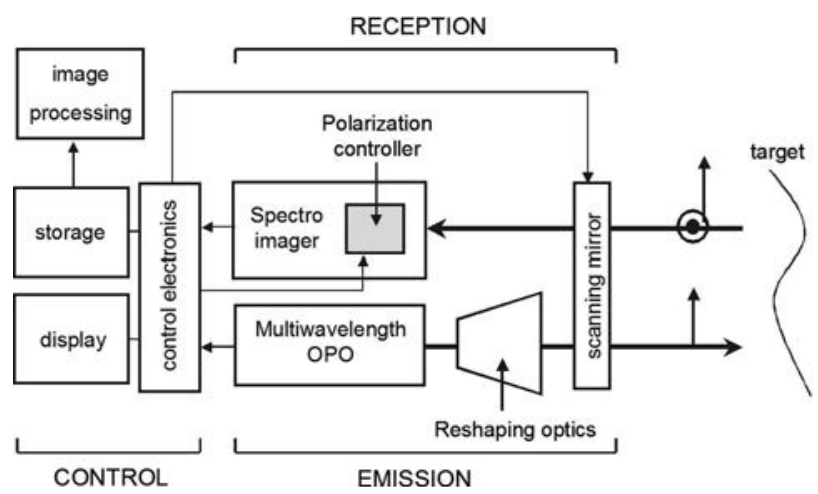

Fig. 1. Building blocks of the active polarimetric and multispectral imager. 


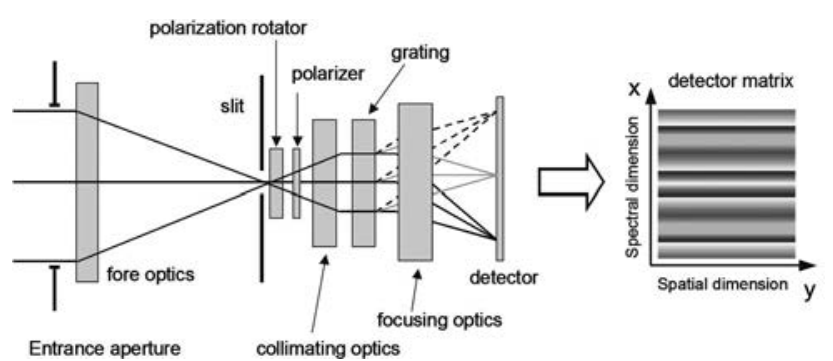

Fig. 2. Left-hand side: hyperspectral imager operating principle. Parallel and crossed polarization analysis is provided by a PLZTbased polarization rotator followed by a linear polarizer. Right-hand side: illustration of single shot image (on the two two-dimensional detector array) when the scene is uniform. This image corresponds, along the $x$ axis, to one spatial dimension of the scene (a line) imaged at different wavelengths along the $y$ axis. The final image is reconstructed after scanning the scene.

detector array corresponds to one spatial dimension of the scene at a different wavelength. This spatial dimension is, actually, a vertical line of the scene. The second spatial dimension is acquired by scanning the scene along the horizontal direction (push broom mode). In other words, each column of the pixel array contains the spectral profile of one point of the scene. Much care has been taken in designing the optical system in order to minimize the point spread function for all wavelengths and all fields of view, as well as to minimize other distortion effects inherent to imaging spectrometers such as smile effect and spectral keystone effect. In the HySpex imaging spectrometers, such distortions are limited to a very small percentage of a pixel and are thus negligible in practice.

A polarization control module, inserted in the hyperspectral imager near the slit, performs linear polarization analysis. The architecture of this module is detailed hereafter. The hyperspectral imager has been optimized to cover the spectral range of 800 $2100 \mathrm{~nm}$. Its spectral resolution is $5 \mathrm{~nm}$. In passive mode operation, 256 spectral channels are available. Since this spectrometer images a vertical line of the scene, it has a large field of view (FOV) along the vertical direction $\left(240 \mathrm{mrad}\right.$, i.e., $\left.14^{\circ}\right)$ and a narrow FOV along the horizontal dimension $(0.75 \mathrm{mrad})$. According to the spectral range we want to address, the detector array used in the system is either a $\mathrm{HgCdTe}$ sensor or an InGaAs sensor. With the spectral responsivity of the latter being limited to $1.7 \mu \mathrm{m}$, it does not cover the entire available spectrum of $800-2100 \mathrm{~nm}$. Nevertheless this sensor is very compact and easy to use and can be sufficient in most cases. Both detector arrays ( $\mathrm{HgCdTe}$ and InGaAs) have resolutions of $320 \times 256$, leading to an instantaneous FOV per pixel of $0.75 \mathrm{mrad} \times 0.75 \mathrm{mrad}$. As previously mentioned, this spectro-imager has push broom architecture and requires the scene to be scanned along the horizontal direction. In groundbased settings this is achieved thanks to a flat mirror mounted on a high-precision motorized step-by-step rotation stage. Figure $\underline{3}$ represents an image of an

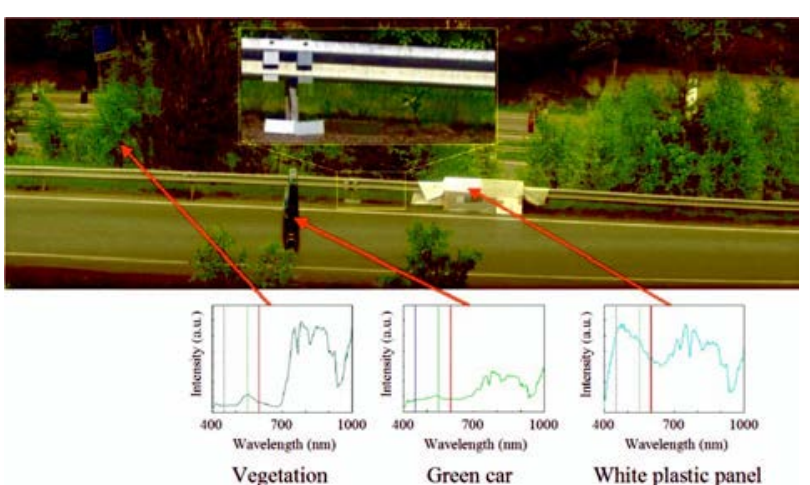

Fig. 3. Example of INT passive image acquired with the HySpex hyperspectral imager. The RGB image has been obtained by merging three spectral channels: $600 \mathrm{~nm}$ (red), $550 \mathrm{~nm}$ (green), and $450 \mathrm{~nm}$ (blue). The spectrum related to each pixel is contained in the third dimension of the final image.

outdoor scene obtained with such a setup, with some examples of spectra.

\section{B. Polarization Analysis Module}

The polarization analysis module is implemented into the hyperspectral imager in the vicinity of the slit. This module consists of a lead lanthanum zirconium titanium (PLZT)-based ceramic plate, which switches the polarization from parallel to crossed with respect to the illumination polarization, followed by a Versalight wire-grid linear polarizer. We used a $\left(\mathrm{Pb}_{1-x} \mathrm{La}_{x}\right)\left(\mathrm{Zr}_{1-y} \mathrm{Ti}_{y}\right)_{1-x / 4} \mathrm{O}_{3}$ ceramic material (PLZT) with (La:Zr:Ti) composition of $(9,65,35)$. This material exhibits a strong Kerr effect [26], with an isotropic electro-optic tensor, meaning that when an electric field, $\vec{E}$, is applied to this material, a change of refractive index occurs along $\vec{E}$. Thus when orienting $\vec{E}$ at $45^{\circ}$ with respect to the incident polarization, one obtains a half-wave plate that flips the polarization from the vertical to the horizontal direction. When followed by a linear polarizer, the assembly acts as a linear polarizer whose orientation is electrically flipped from $0^{\circ}$ to $90^{\circ}$.

Our choice of PLZT ceramic for the realization of the polarization switch was motivated by the following reasons. First, PLZT has a very low linear absorption, typically lower than $0.04 \mathrm{~mm}^{-1}$, in the spectral range covered by the hyperspectral imager, as shown in Fig. 4. Second, its electro-optic coefficient (typically $3-5 \times 10^{-16} \mathrm{~m}^{2} / \mathrm{V}^{2}$ ) is very high and almost constant over the spectral range of interest (see Fig. 4). Third, its response time has been measured to be in the microsecond range and is in practice limited by the capacitance effect due to the presence of the electrodes. These characteristics make PLZT a very good candidate for broad bandwidth very near-infrared and short-wave infrared applications.

In order to optimize the overlap between the applied electric field and the optical wave in the electro-optic material, we buried the electrodes into the PLZT material [27] (see Fig. 5). Indeed when surface electrodes are used, first, the penetration depth of the electric field inside the material is limited, and 


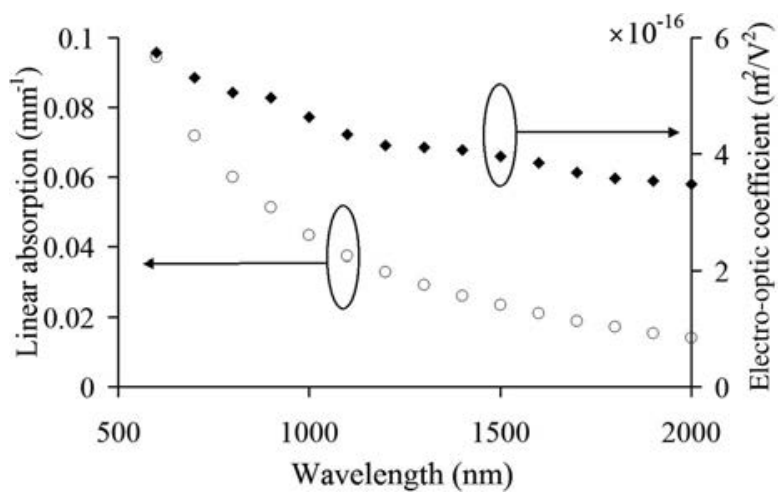

Fig. 4. Linear absorption and electro-optic coefficient of the PLZT ceramic, used for polarization switching, as a function of wavelength.

second, high local electric fields appear in the vicinity of the electrodes, leading to electric-field-induced scattering losses in the ceramic due to the piezoelectric dilatation of the ceramic grains. Burying the electrodes in the PLZT ceramic produces a homogeneous electric field along the total thickness of the plate. Consequently point effects are avoided, thus significantly limiting the electric-field-induced scattering. Compared to the surface electrodes arrangement (deposited on both sides of the plate), using the full thickness of the material leads to an increase of the resulting birefringence by a factor $e / d$, where $e$ is the plate thickness, and $d$ is the interelectrodes gap. The electro-optic birefringence being proportional to the square root of the applied voltage, this enables a reduction of the applied voltage by approximately a factor of 2 . Basically the required voltage to rotate the polarization by $90^{\circ}$ is of approximately $50 \mathrm{~V}$ when the spatial separation between the electrodes is $100 \mu \mathrm{m}$.

\section{Emission Part}

A multiwavelength optical parametric oscillator (OPO) generates the illumination beam. The OPO gain medium is a custom periodically poled $\mathrm{MgO}-$ doped lithium niobate crystal (PPMgLN) fabricated with four adjacent gratings [28]. The periods of these (a)

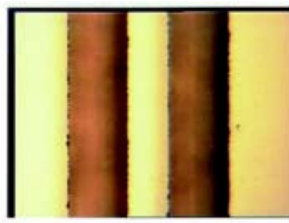

(b)

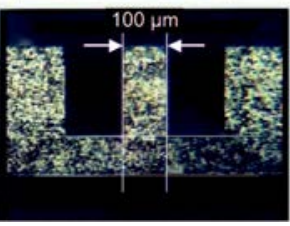

(c)

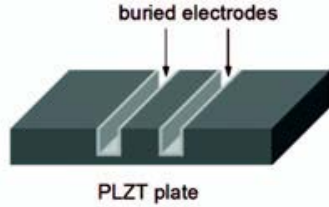

(d)

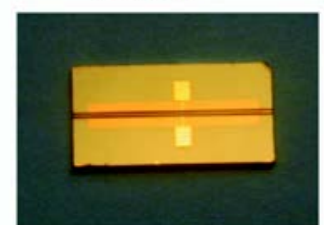

Fig. 5. (a) Top and (b) side microscope views of the processed PLZT polarization rotator showing the parallel grooves receiving the electrodes. (c), (d) Drawing and photograph, respectively, of the PLZT polarization rotator plate with its contacts. gratings have been optimized to obtain phase matching at the wavelengths of interest. Each grating potentially provides two wavelengths: the "signal" and the "idler." Nevertheless, from a practical point of view, only the signal wavelengths are exploited in the experiment, the idler wavelengths being out of the imager spectral range $(800-2100 \mathrm{~nm})$. Consequently five wavelength channels are available: four generated by the OPO itself, and the fifth one corresponding to the remaining fraction of unconverted pump.

The active medium is inserted into an optical cavity formed by two plane mirrors and pumped by an elliptical beam. This architecture enables a multiwavelength output on a single beam. The OPO is pumped with a pulsed Nd:YLF laser (DIVA from THALES Laser, France). This laser provides, at $1064 \mathrm{~nm}$, a $10 \mathrm{~ns}$ duration and $40 \mathrm{~mJ}$ energy pulses at a repetition rate of $10 \mathrm{~Hz}$. The pump beam is elliptically reshaped in order to efficiently pump all the adjacent gratings of the OPO crystal. The large effective nonlinear coefficient of lithium niobate used in quasi-phase-matched configuration makes it possible to extract significant output energy, typically $1 \mathrm{~mJ}$ per channel. It is worthwhile to notice that the OPO architecture is optimized in order to provide, at each channel, a sufficiently large linewidth to get rid of speckle noise in active images [29] (see the images reported in Section 3). Typical linewidths of a few nanometers have been obtained, leading to a coherence length lower than $1 \mathrm{~mm}$. For instance, the spectral shape of channel 3, reported in Fig. 6, shows a linewidth of $6.5 \mathrm{~nm}$.

The beam coming out from the OPO is directed to an optical reshaping module that matches the illumination beam divergence with respect to the imager FOV in both the $x$ and the $y$ directions (see Subsection 2.A). With the hyperspectral imager being designed to operate in the push broom configuration, the illumination beam is sent onto the step-by-step scanning mirror (see Subsection 2.A). Thus one ensures a good overlap between the illumination beam

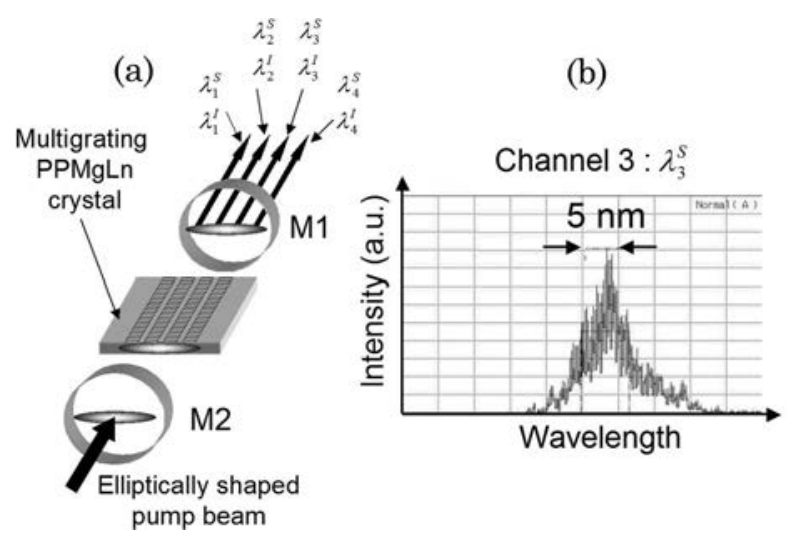

Fig. 6. (a) Drawing of the multiwavelength OPO used as illumination source. M1 and M2 are the cavity mirrors. (b) Typical spectral shape of one wavelength channel. The large spectral width of each channel enables us to obtain active images free of speckle. 
and the line of sight during image acquisition. In summary the far field illumination beam consists of a vertical straight line composed of light at five different wavelengths and scans the scene along the horizontal direction.

Obviously the number of illuminating wavelengths and their values must be chosen in accordance with the nature of the objects we want to detect and the complexity of the scene under consideration (no uniform backgrounds). It must be noticed that exploiting the high spectral resolution can be useful in many situations. For instance, in real-field remote imaging, high spectral resolution is of great interest for detecting objects whose dimensions in the image are smaller than the detector pixel size (subresolution detection). It is also a good approach when the object under consideration has a well-known spectral singularity as compared to the surrounding background. In the situation where the object size is larger than the pixel size, and in the context of anomaly detection, we have shown that only a few wavelengths are necessary to obtain efficient detection [30]. Indeed the spectral signatures of materials most frequently encountered in remote sensing applications have smooth variations within the atmospheric transmission bands. Consequently a high spectral resolution is not necessary provided that the target covers at least a few pixels in the image. In these conditions using too many spectral channels may even reduce the detection performance [30]. Indeed practical detectors must estimate some parameters from the data. Their number is at least proportional to the number of spectral channels. If the extra spectral channels do not bring enough information to compensate for the extra nuisance parameters, target detection performance may decrease. Moreover, since the computation complexity of the detection algorithm is at least proportional to the number of spectral channels, reducing their number is attractive.

\section{Control and Acquisition Part}

The general operation scheme of the laboratory demonstrator is shown in Fig. 1. Let us recall that the reception part consists of a push broom hyperspectral imager, in which we have implemented a polarization analysis module (see detailed descriptions in Subsections 2.A and 2.B). The polarization analysis module allows acquisition of two different images of the scene, one image being formed with light polarized parallel to the illumination state and the other image being formed with light polarized in the orthogonal state. The illumination source is the linearly polarized pulsed multiwavelength OPO described in Subsection 2.C.

In order to get both active and passive images, the camera is synchronized at twice the pulse repetition frequency, namely, at $20 \mathrm{~Hz}$. Active and passive images are thus interleaved. This offers the possibility to get rid of the ambient illumination by subtracting the passive image from the active one.
Each acquisition shot forms on the camera sensor an image related to a vertical line of the scene at different wavelengths. The acquisition sequence is as follows. For each position of the scanning mirror, four images are acquired. The first two images, $I_{\|}^{\text {act }}(x, \lambda)$ and $I_{\|}^{\mathrm{pas}}(x, \lambda)$ are formed with light parallel to the illumination state, respectively, under active and passive illumination. The polarization state is then rotated to acquire a couple of images, $I_{\perp}^{\text {act }}(x, \lambda)$ and $I_{\perp}^{\text {pas }}(x, \lambda)$, corresponding to the crossed state of polarization, respectively, under active and passive illumination. For each polarization state the passive image is subtracted from the active image in order to get pure active images devoid of ambient light. These images will be denoted $I_{\|}(x, \lambda)$ and $I_{\perp}(x, \lambda)$ :

$$
\begin{aligned}
& I_{/ /}(x, \lambda)=I_{\|}^{\mathrm{act}}(x, \lambda)-I_{\|}^{\mathrm{pas}}(x, \lambda), \\
& I_{\perp}(x, \lambda)=I_{\perp}^{\mathrm{act}}(x, \lambda)-I_{\perp}^{\mathrm{pas}}(x, \lambda) .
\end{aligned}
$$

Finally the INT image is simply computed through the following relation:

$$
\operatorname{INT}(x, \lambda)=I_{\|}(x, \lambda)+I_{\perp}(x, \lambda),
$$

and the polarimetric image is given by

$$
\operatorname{OSC}(x, \lambda)=\frac{I_{\|}(x, \lambda)-I_{\perp}(x, \lambda)}{I_{\|}(x, \lambda)+I_{\perp}(x, \lambda)}
$$

The final image is constructed by scanning the scene along the second spatial dimension (see Subsection 2.C). As a result we end up with four three-dimensional images: active INT, passive INT, active OSC, and passive OSC, in which the two first dimensions correspond to the two spatial dimensions of the scene, meanwhile the third dimension corresponds to wavelength. The calibration of the imager takes into account the spatial and spectral nonuniform response of the pixels as well as the hyperspectral imager's transmission function and, finally, the wavelength dependence of the polarization rotation function. It is worthwhile to notice that the voltage, at which a $90^{\circ}$ polarization rotation occurs, is wavelength dependent. Ideally one needs to set to the PLZT ceramic a different voltage value for each wavelength. This is not compatible with the operation principle of the hyperspectral imager, which records all the wavelengths simultaneously. That is, the voltage $V_{0}$ applied to the PLZT ceramic is chosen in order to get a perfect rotation of the polarization at a well-established wavelength. This wavelength, lying close to the middle of the spectrum of interest, is computed so that minimum postcorrection is required over the rest of the spectrum. From a practical point of view, one uses a large spectral width polarized source based on a blackbody followed by a grid polarizer. The grid polarizer is oriented along the parallel direction, and two images are acquired with and without applying $V_{0}$ to the ceramic. Then the same sequence is acquired orienting the 
polarizer along the orthogonal direction. These four images, which form the basis of our polarization calibration procedure, are finally processed in order to extract the calibration parameters. Such a method takes into account the nonperfect rotation of the polarization with respect to wavelength and also all the residual artifacts, including those linked to the imager optics such as retardance and diattenuation. Moreover, real-time correction of the image during acquisition is also performed in order to take into account the energy fluctuations of the illuminating pulses. A photograph of the laboratory demonstrator is shown in Fig. 7 .

\section{Application to Target Detection}

Here we present some results that illustrate the advantage of simultaneously acquiring INT and OSC images for target detection. We first briefly describe the noise model and the detection algorithms used. We then illustrate the added value brought by performing joint multispectral INT and polarimetric imaging.

\section{A. Detection Algorithm and Performance Estimation}

As said in Subsection 2.C, the illuminating laser was designed to have a large linewidth in order to get rid of speckle noise. We checked that, in real images, speckle noise was actually not the dominant source of perturbation in this system. For that purpose we divided the image of a scene in small square regions of $16 \times 16$ pixels in size. We estimated the mean and the standard deviation of the pixels in each of these regions, and we checked that the standard deviation was independent of the mean. Since these perturba-

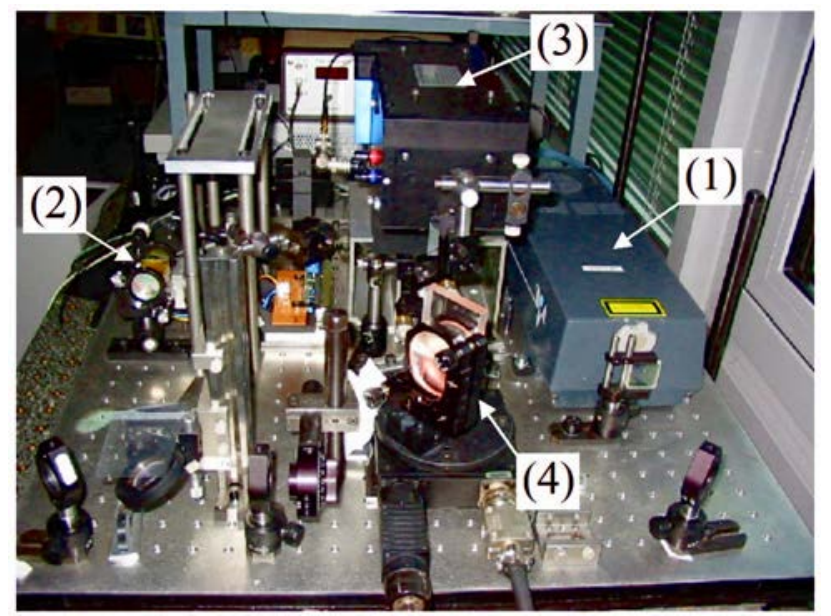

Fig. 7. Overview of the active polarimetric and multispectral imager compacted on $60 \mathrm{~cm} \times 60 \mathrm{~cm}$ breadboard. The pump laser in gray appears on the right (1). After beam reshaping, this laser feeds a multiwavelength OPO appearing on the left of the view (2). The OPO output beam is polarized and carries different discrete wavelengths. It is reshaped underneath (3) in order to shine the scene with a vertical straight line matching the FOV of the hyperspectral imager. The hyperspectral imager appears in the middle of the view with a black housing (3). The wire entering the imager controls the PLZT-based polarimetric module. The scanning mirror labeled (4) acts for both emission and reception. tions are mainly independent from the useful signal, we chose to model the noise that perturbs the images as additive and Gaussian. We are interested in detecting small but resolved targets. To perform detection we used the generalized likelihood ratio test (GLRT) detection algorithm [31], which consists of scanning the scene with a mask, F, composed of two parts: $w$, which represents the shape of the target, and $\bar{w}$, which is the complementary of $w$ in $\mathbf{F}$. If one is in the presence of additive Gaussian noise with variance $\sigma_{k}^{2}$ in a channel $k$ ( $k$ being related to wavelength or to INT/OSC in our case), it can be shown that the GLRT consists of computing, at each location, $(i, j)$ of the mask $\mathbf{F}$ in the image:

$$
R(i, j)=\frac{N_{w} N_{\bar{w}}}{N_{w}+N_{\bar{w}}} \frac{\left(\hat{m}_{w}^{k}-\hat{m}_{\bar{w}}^{k}\right)^{2}}{\sigma_{k}^{2}},
$$

where $N_{\bar{w}}$ is the number of pixels in $w, N_{\bar{w}}$ is the number of pixels in $\bar{w}$, and $\hat{m}_{w}^{k}$ and $\hat{m}_{\bar{w}}^{k}$ denote the empirical mean estimated in $w$ and $\bar{w}$, respectively. One then makes a decision by choosing between the two following hypotheses:

$$
\begin{aligned}
& -\mathbf{H}_{\mathbf{1}} \text { : detect a target at position }(i, j) \text { if } R(i, j)> \\
& \lambda \text { or } \mathbf{H}_{0} \text { : decide that there is no target at posi- } \\
& \text { tion }(i, j) \text {. }
\end{aligned}
$$

The most computation intensive part of this algorithm consists of computing moving averages in masks $w$ and $\bar{w}$. This is equivalent to matched filtering and can be performed efficiently with fast Fourier transforms. Of course this detection method is very simplistic. It assumes that the size of the target is known, and it is prone to false alarms. However, we make use of the detection method mainly to estimate a local contrast around the target and to be able to compare this contrast in the different INT/OSC and wavelength channels.

It is important to have a quantitative evaluation of the quality of the detection algorithms in each channel. The standard way of doing so is to compute the receiver operating characteristic, which necessitates many different realizations of the scene. In a real image there is only a single realization. Consequently, in the present case, one can only rely on the $R(i, j)$ plane that has been obtained from the image in order to estimate the quality of the detection result. The computation of $R(i, j)$ at each point of the scene yields an image in which high values correspond to high probability that a target is present at this position in the scene. This image thus contains a peak at the position of a target. In order to quantify the detection performance, one will thus use the peak-tosidelobe ratio (PSR), which is defined as follows. Let $\left(i_{0}, j_{0}\right)$ represent the position of the target in the image. A mask, $T$, which is square-shaped and $3 \times 3$ pixels in size, is centered around the target as well as the mask, $B$, whose shape is represented 


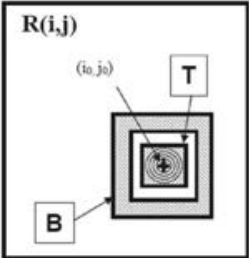

Fig. 8. Representation of the regions $T$ (target) and $B$ (background) involved in the definition of the PSR, which is defined in Eq. (7).

in Fig. 8. The PSR is defined as follows:

$$
\operatorname{PSR}=10 \log _{10}\left[\frac{\left(\hat{m}_{T}-\hat{m}_{B}\right)^{2}}{\hat{\sigma}_{B}^{2}}\right],
$$

where

$$
\begin{aligned}
& \hat{m}_{T}=\frac{1}{N_{T}} \sum_{(i, j) \in T} R(i, j), \quad \hat{m}_{B}=\frac{1}{N_{B}} \sum_{(i, j) \in B} R(i, j), \\
& \hat{\sigma}_{B}^{2}=\frac{1}{N_{B}} \sum_{(i, j) \in T}\left[R(i, j)-\hat{m}_{B}\right]^{2},
\end{aligned}
$$

and $N_{T}\left(N_{B}\right)$ denotes the number of pixels in mask $T(B)$.

The PSR quantifies the "conspicuousness" of the peak corresponding to the position of the target in the $R(i, j)$ plane. It is clear that the higher the PSR, the better the performance. The PSR is used in the following to compare the detection quality in the different channels.

\section{B. Detection Results}

In order to evaluate the performance of the different imaging modes, we used as targets small painted metallic plates located $20 \mathrm{~m}$ away from the imaging system and appearing against different types of background, such as foliage, grass, sand, or soil. For example, Fig. $\underline{9}$ depicts a small metallic plate

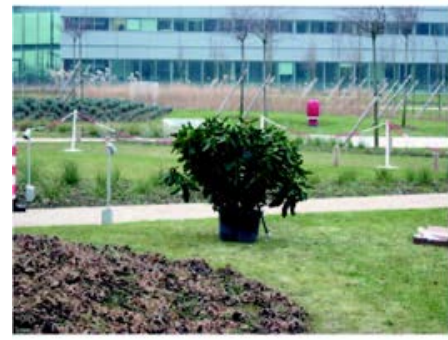

(a)

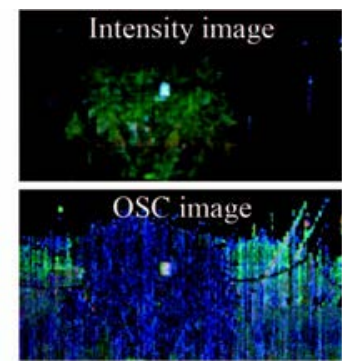

(b)
Fig. 9. (a) View (photograph in the visible domain) of a green painted panel positioned on a foliage background. The panel is difficult to see in the visible domain. (b) Demonstrator acquisition: RGB-coded INT and polarimetric (OSC) images in the near-infrared obtained by merging channels $\lambda_{1}^{S}, \lambda_{2}^{S}$, and $\lambda_{3}^{S}$. Here the target appears on both the INT and the OSC images. The OSC image offers a uniform background more suitable for anomaly detection. covered with green paint appearing on green foliage with visible passive imaging and the corresponding INT and OSC active images in the near-infrared. The two latter images are in false color and have been built by coding in RGB three images obtained at three different wavelengths $\left\{\lambda_{1}, \lambda_{2}, \lambda_{3}\right\}$ in the range $800-2100 \mathrm{~nm}$. It is seen that the target appears with a better contrast in the OSC image. Moreover, it can be noticed that the background surrounding the target is much more homogeneous in this image. This is mainly due to two factors. First, the OSC is, by construction, independent of the inhomogeneity of the illuminating beam [32]. Second, the degree of polarization is less dependent on the illumination orientation than the INT reflectance. Since the leaves of the plant are randomly oriented with respect to the direction of illumination, they create a textured background in the INT image, whereas it is much more uniform in the OSC image.

It is also interesting to separately consider the images obtained at different wavelengths. We display in Fig. 10 the INT and OSC images at the three wavelengths, $\left\{\lambda_{1}, \lambda_{2}, \lambda_{3}\right\}$, together with the corresponding $R(i, j)$ detection planes [see Eq. (6)]. It is clearly seen that, at wavelength $\lambda_{1}$, the target is easily detected in the OSC image, whereas it is not in the INT image. On the other hand, at wavelength $\lambda_{3}$, detection is easier in the INT image, and the detection peak is "cleaner" than in the OSC image. This is confirmed in Fig. 11, where we have represented the value of the PSR in the INT and OSC detection planes. The OSC and INT images have clearly "complementary" behaviors since, in OSC images, the PSR decreases with wavelength, whereas, in INT images, it tends to increase. This is coherent with a recently proposed model of INT and OSC behaviors of diffuse materials [13]. Using the Kubelka-Munk theory [33], this model shows that, in the case of diffuse objects, when the reflected INT increases,

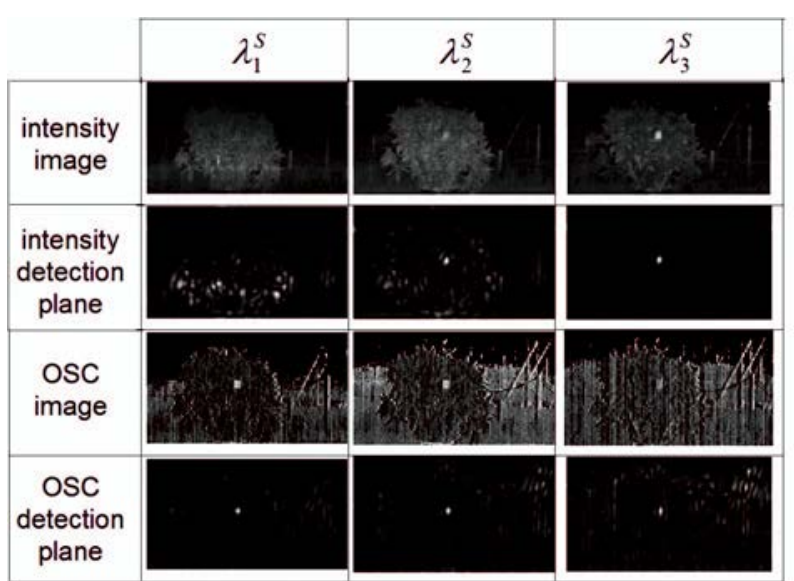

Fig. 10. Same scene as in Fig. 9 observed with the active system in three wavelengths and the corresponding GLRT detection planes. First row: INT image; second row, GLRT detection plane obtained from the INT images; third row, OSC image; and fourth row, GLRT detection plane obtained from the OSC image. 


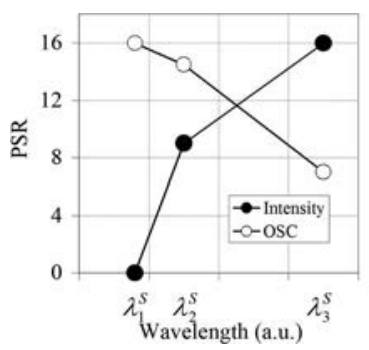

Fig. 11. Values of the PSR for the three considered wavelengths $\left(\lambda_{1}^{S}, \lambda_{2}^{S}\right.$, and $\left.\lambda_{3}^{S}\right)$ in the INT and OSC detection planes.

the OSC of the reflected light tends to decrease. It is worth emphasizing that, when operating a polarimetric imaging system, one also acquires the INT image so that both pieces of information are available. The results in Figs. 10 and 11 show that the joint knowledge of the INT and OSC images at three wavelengths enables one to obtain a significant PSR, thus a good detection performance, for all wavelengths. This is thus a good example of the usefulness of an imager that is both multispectral and polarimetric.

Besides providing useful contrast, OSC imaging also possesses interesting invariance properties to surface orientation. The image in Fig. 12 represents an outdoor scene with two metallic plates appearing on a grass background. The metallic plates are of the same size and are covered with the same green paint. The plate on the left side lies on the ground, so its surface makes a quite oblique angle with the line of sight. On the other hand, the plate on the right-hand side is vertical, and its surface is quasiorthogonal to the line of sight. In the INT image the plate on the right-hand side is well visible, but the one on the left-hand side is not since its INT reflectivity highly depends on its orientation. On the other hand, in the OSC image the two plates are well visible and can be easily detected with the GLRT algorithm. This example again illustrates the fact that the OSC response is significantly less dependent on the orientation than the INT response.

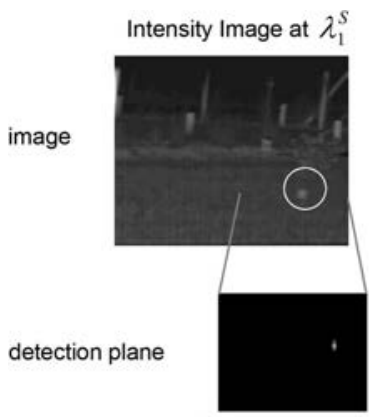

(a)

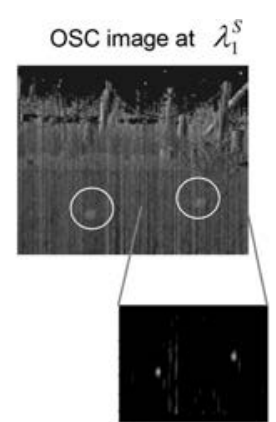

(b)
Fig. 12. Detection of two identical green plates appearing on a grass background. The first plate (on the left) is lying on the grass, while the second one (on the right) is positioned perpendicular to the floor. The detection algorithm applied to the INT image allows only the second plate to be detected. When the algorithm is applied to the OSC image, the two plates are detected.

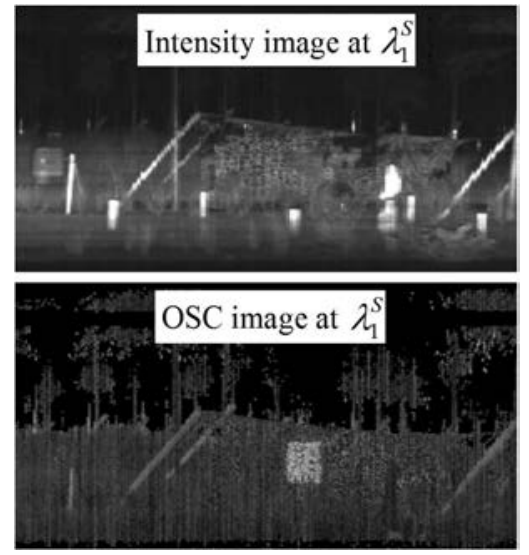

Fig. 13. Decamouflage of a metallic panel hidden by a camouflage net.

Finally we represent in Fig. 13 an outdoor scene with a large sand-blasted metallic plate located approximately $40 \mathrm{~m}$ from the imager. It is hidden by a camouflage net located in front of it and is not seen in the INT image. On the other hand, it appears with a high contrast in the OSC image. The reason is that the part of the light that goes through the mesh of the camouflage net and that is backscattered by the metallic plate remains perfectly polarized. As a result its OSC is close to 1 . Even if the spatial resolution of the imager is lower than the dimensions of the camouflage net mesh, the averaged OSC in the region where the metallic plate is located will be higher than that of the camouflage net and the surrounding background.

\section{Conclusion}

A compact laboratory demonstrator providing, at the same time, active polarimetric and multispectral images has been realized. The reception part of this demonstrator consists of a hyperspectral imager covering the spectral range $800-2100 \mathrm{~nm}$. Polarization control of the received light is achieved thanks to a fast PLZT-based polarization rotator implemented inside the hyperspectral imager. At the emission side a PPMgLN OPO has been designed to provide polarized optical pulses simultaneously carrying several wavelengths. The imaging system has been exploited in view of enhancing the contrast between man-made objects and natural backgrounds.

We show that the spectral/polarimetric function brings additional information on the scene content, especially when polarimetric images are interpreted in conjunction with INT images. Indeed the INT image and its counterpart polarimetric image are found to be complementary in most cases. This typical property of diffuse objects makes it possible to enhance the detection sensitivity of low-contrast targets, provided that the illumination wavelengths are properly chosen.

The authors acknowledge the Délégation Générale pour l'Armement (France) and Forsvarets ForskningsInstitutt (Norway) for supporting this work. 


\section{References}

1. B. Javidi, S. H. Hong, and O. Matoba, "Multidimensional optical sensor and imaging system," Appl. Opt. 45, 29862994 (2006).

2. D. Kim, C. Warde, K. Vaccaro, and C. Woods, "Imaging multispectral polarimetric sensor: single-pixel design, fabrication, and characterization," Appl. Opt. 42, 3756-3764 (2003).

3. C. A. Farlow, D. B. Chenault, J. L. Pezzaniti, K. D. Spradley, and M. G. Gulley, "Imaging polarimeter development and applications," Proc. SPIE 4481, 118-125 (2002).

4. J. Chowdhary, B. Cairns, and L. D. Travis "Contribution of water-leaving radiances to multiangle, multispectral polarimetric observations over the open ocean: bio-optical model results for case 1 waters," Appl. Opt. 45, 5542-5567 (2006).

5. F. A. Sadjadi and C. S. Chun, "New experiments in the use of infrared polarization in the detection of small targets," Proc. SPIE 4379, 144-155 (2001).

6. G. Miecznik, R. Illing, S. Petroy, and I. N. Sokolik, "Sensitivity metric approach for retrieval of aerosol properties from multiangular and multispectral polarized radiances," Appl. Opt. 44, 4186-4204 (2005).

7. S. R. Loe and M. Duggin, "Hyperspectral imaging polarimeter design and calibration," Proc. SPIE 4481, 195-205 (2001).

8. J. S. Tyo and T. S. Turner, "Imaging spectropolarimeters for use in visible and infrared remote sensing," Proc. SPIE 3753, 214-224 (1999).

9. J. L. Denes, M. S. Gottlieb, B. Kaminsky, and D. F. Huber, "Spectropolarimetric imaging for object recognition," Proc. SPIE 3240, 8-18 (1998).

10. H. E. Scott, S. H. Jones, F. J. Iannarilli, and K. Annen, "Hyperspectral IR polarimetry with applications in demining and unexploded ordnance detection," Proc. SPIE 3534, 300 320 (1998)

11. L. Le Hors, P. Hartemann, and S. Breugnot, "Multispectral polarization active imager in the visible band," Proc. SPIE 4035, 380-389 (2000).

12. L. Le Hors, P. Hartemann, D. Dolfi, and S. Breugnot, "A phenomenological model of paints for multispectral polarimetric imaging," Proc. SPIE 4370, 94-105 (2001).

13. M. Alouini, F. Goudail, N. Roux, L. Le Hors, P. Hartemann, S. Breugnot, and D. Dolfi, "Active spectro-polarimetric imaging: signature modeling, imaging demonstrator and target detection," Eur. Phys. J. Appl. Phys. 42, 129-139 (2008).

14. A. D. Portnoy, M. E. Gehm, and D. J. Brady, "Pushbroom hyperspectral imaging with a coded aperture," in Frontiers in Optics, OSA Technical Digest (CD) (Optical Society of America, 2006), paper FMB2.
15. C. R. Howle, D. J. M. Stothard, C. F. Rae, M. Ross, B. S. Truscott, C. D. Dyer, and M. H. Dunn, "Active hyperspectral imaging system for the detection of liquids," Proc. SPIE 6954, 69540L (2008).

16. E. Collet, Polarized Light (Marcel Deckker, 1993).

17. S. Manhas, M. K. Swami, P. Buddhiwant, N. Ghosh, P. K. Gupta, and J. Singh, "Mueller matrix approach for determination of optical rotation in chiral turbid media in backscattering geometry," Opt. Express 14, 190-202 (2006).

18. K. M. Twietmeyer, R. A. Chipman, A. E. Elsner, Y. Zhao, and D. VanNasdale, "Mueller matrix retinal imager with optimized polarization conditions," Opt. Express 16, 2133921354 (2008).

19. C. Brosseau, Polarized Light (Wiley, 1998).

20. B. Laude-Boulesteix, A. De Martino, B. Drévillon, and L. Schwartz, "Mueller polarimetric imaging system with liquid crystals," Appl. Opt. 43, 2824-2832 (2004).

21. S. Y. Lu and R. A. Chipman, "Interpretation of Mueller matrices based on polar decomposition," J. Opt. Soc. Am. A 13, 1106-1113 (1996).

22. P. Eliès, B. Le Jeune, M. Floc'h, and J. Lotrian, "Depolarization classification of metallic and dielectric targets," Proc. SPIE 3059, 174 (1997).

23. S. Breugnot and P. Clémenceau, "Modeling and performances of a polarization active imager at $806 \mathrm{~nm}$," Opt. Eng. 39, 26812688 (2000).

24. S. Huard, Polarization of Light (Wiley, 1997).

25. http://www.neo.no/.

26. G. H. Haertling, "PLZT electrooptic materials and applications, a review," Ferroelectrics 75, 25-55 (1987).

27. J. Bourderionnet, D. Dolfi, J. P. Huignard, B. Ea-Kim, E. Corbel, D. Purer, A. Poudoulec, and D. Gauden, "Endless fiber-to-fiber polarization controller based on ceramic programmable waveplates," IEEE Photonics Technol. Lett. 16, 1101-1103 (2004).

28. http://www.hcphotonics.com/.

29. M. Alouini, F. Goudail, P. Refregier, A. Grisard, E. Lallier, and D. Dolfi, "Multispectral polarimetric imaging with coherent illumination: towards higher image contrast," Proc. SPIE 5432, 133-144 (2004)

30. F. Goudail, N. Roux, I. Baarstad, T. Løke, P. Kaspersen, M. Alouini, and X. Normandin, "Some practical issues in anomaly detection and exploitation of regions of interest in hyperspectral images," Appl. Opt. 45, 5223-5236 (2006).

31. S. M. Kay, Fundamentals of Statistical Signal Processing: Detection Theory, Vol. 2 (Prentice-Hall, 1998).

32. F. Goudail and P. Réfrégier, "Statistical techniques for target detection in polarization diversity images," Opt. Lett. 26, 644646 (2001).

33. P. Kubelka and F. Munk, "Ein Beitrag zur Optik der Farbanstriche," Z. Tech. Phys. 12, 593-601 (1931). 